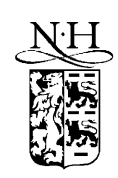

\title{
Integrability and exact solution for coupled BCS systems associated with the $s u(4)$ Lie algebra
}

\author{
X.-W. Guan ${ }^{\text {a }}$, A. Foerster ${ }^{\mathrm{a}, \mathrm{b}}$, J. Links $^{\mathrm{c}}$, H.-Q. Zhou ${ }^{\mathrm{c}}$ \\ ${ }^{a}$ Instituto de Fisica da UFRGS, Av. Bento Goncalves, 9500, Porto Alegre, 91501-970, Brazil \\ b Institut für Theoretische Physik, der FU-Berlin, Arnimallee 14, Berlin, Germany \\ c Centre for Mathematical Physics, School of Physical Sciences, The University of Queensland, 4072, Australia
}

Received 13 May 2002; received in revised form 9 August 2002; accepted 26 August 2002

\begin{abstract}
We introduce an integrable model for two coupled BCS systems through a solution of the YangBaxter equation associated with the Lie algebra $s u(4)$. By employing the algebraic Bethe ansatz, we determine the exact solution for the energy spectrum. An asymptotic analysis is conducted to determine the leading terms in the ground state energy, the gap and some one point correlation functions at zero temperature.
\end{abstract}

(C) 2002 Published by Elsevier Science B.V.

PACS: $71.24 .+\mathrm{q} ; 74.20 . \mathrm{Fg}$

Keywords: BCS model; Cooper pair; Quantum $R$-matrix; Ground state

\section{Introduction}

The role of the Yang-Baxter equation in the study of quantum mechanical models has a long and distinguished history. Notable examples are the $X Y Z$ chain [1], the $t-J$ model at supersymmetric coupling [2] and the Hubbard model [3], each of which is both integrable and exactly solvable as a result of the formulation for each model through the Quantum Inverse Scattering Method (QISM). The key concept of the QISM is the notion of mutually commuting transfer matrices, the existence of which is a result of the YangBaxter equation. In each of the above examples the Hamiltonian of the model is defined as the logarithmic derivative of the transfer matrix, and by the nature of the construction this yields a model defined on a one-dimensional lattice with nearest neighbour interactions,

E-mail address: guan@if.ufrgs.br (X.-W. Guan). 
where it is possible for both integrability and solvability to hold for a variety of boundary conditions.

The application of the QISM however can be applied on a much more general level. In the context of the present work, it is appropriate to mention, for example, the work of Gaudin [4] in relation to the construction of systems with long range interactions. Very closely related to Gaudin's Hamiltonians is the BCS model, the exact solution of which was quite remarkably found in 1963 by Richardson [5], while integrability was established much later by Cambiaggio et al. in 1997 [6]. The exact solution of the BCS model has come under close scrutiny in recent years due to its application in the theory of metallic nanograins [7]. Specifically, the experiments of Ralph, Black and Tinkham [8] have shown that it is not valid to apply the BCS mean field theory for systems of nanoscale size. As a result, one has to turn to the exact solution of Richardson in order to conduct a reliable analysis. However, the approaches adopted in [5,6] make no reference to the Yang-Baxter equation or the QISM (indeed QISM was not developed until many years after Richardson's work), and since historically the two facets of integrability and solvability have been intimately linked in the QISM framework, it was a natural question to ask whether the BCS model could be recast through this technique. An affirmative answer was given in $[9,10]$ with the surprising result that the $R$-matrix solution of the Yang-Baxter equation which is needed in the construction of the BCS Hamiltonian is one of the simplest known examples, being that associated with the $s u(2)$ algebra. Given that a great volume of literature exists devoted to solutions of the Yang-Baxter equation associated with representations of simple Lie algebras, there is a vast opportunity to investigate generalized models. An important step towards this has already been achieved in [11] where a connection has been established between Chern-Simons theory and integrability of models associated with an arbitrary Lie algebra, which is achieved through the Knizhnik-Zamolodchikov-Bernard equations.

Such generalized models can be interpreted as coupled BCS systems, at least in the sense that every simple Lie algebra can be generated by a system of simple roots which each form an $s u(2)$ subalgebra. An example of this was given in [12] where the Lie algebra employed was so(5). In this instance, the model constructed reproduces the one studied by Richardson in 1966 [13] describing proton-proton and neutron-neutron pairing as well as a coupling term for the scattering of proton-neutron pairs. Here we shall introduce a model based on the $s u(4)$ Lie algebra symmetry which can also be interpreted as a nuclear system where there are now different types of pairing interactions. The Hamiltonian takes the form of two BCS systems which individually describe pairing interactions for the protons and neutrons and the scattering of bound proton pairs-neutron pairs, which is in contrast to the proton-neutron pairs of [12]. This interpretation is possible because the number operator for each system provides a good quantum number; i.e., the number operators are conserved. Therefore we can identify each BCS system with a particular distinguishable particle, which in this case are the protons and neutrons. It is worth remarking that this situation is inherently different to the pairing models described in [14] based on higher spin representations of the $s u(2)$ algebra. In these cases, the only good quantum number is the total number of particles in the combined system. There, individual particle numbers are not conserved and thus the models can be interpreted as describing a Josephson tunneling phenomena. 
The paper is organized as follows. In Section 2 we present the construction of the model through the QISM. In Section 3 the exact solution of the model is given by means of the algebraic Bethe ansatz. An analysis of the asymptotic solutions of the Bethe ansatz equations is presented in Section 4, where the ground state energy, the gap in the spectrum of elementary excitations, as well as the derivation of some correlation functions in this asymptotic regime, are presented. A summary of the main results can be found in Section 5.

\section{Coupled pairing Hamiltonian and integrability}

Let us begin by introducing the following Hamiltonian

$$
\begin{aligned}
H= & \operatorname{BCS}(1)+\operatorname{BCS}(2)-g \sum_{j, k}^{\Omega} b_{j}^{+}(1) b_{j}^{+}(2) b_{k}(2) b_{k}(1) \\
& +g \sum_{j, k}^{\Omega} b_{j}^{+}(1) b_{k}(1)\left(n_{j}(2)-n_{k}(2)\right)^{2} \\
& +g \sum_{j, k}^{\Omega} b_{j}^{+}(2) b_{k}(2)\left(n_{j}(1)-n_{k}(1)\right)^{2},
\end{aligned}
$$

where

$$
\operatorname{BCS}(a)=\sum_{j=1}^{\Omega} 2 \epsilon_{j} n_{j}(a)-g \sum_{j, k}^{\Omega} b_{j}^{+}(a) b_{k}(a) .
$$

Above the operators $b_{j}(a), b_{j}^{+}(a)$ are the annihilation and creation operators for the hardcore bosons (or Cooper pairs) in system $a$, and $j$ refers to the single particle energy level with energy $\epsilon_{j}$. We will assume that the values $\epsilon_{j}$ are distinct. Further, $g$ is a coupling strength constant for the scattering of Cooper pairs and $n_{j}(a)=b_{j}^{+}(a) b_{j}(a)$, is the Cooper pair number operator. As in the case of the usual BCS system there is a blocking effect (e.g., see [7]), as there is no scattering of any unpaired states. For each level $j$ there are actually sixteen local states, but the nature of the Hamiltonian means that only on a subspace spanned by four of these states, where there are no unpaired states, is the scattering nontrivial (see (2.7)). Hereafter we will restrict our analysis to this subspace.

On this restricted subspace the operators $b_{j}^{+}(a)=c_{j \uparrow}^{\dagger}(a) c_{j \downarrow}^{\dagger}(a), b_{j}(a)=c_{j \downarrow}(a) c_{j \uparrow}(a)$, where $c_{j \sigma}, c_{j \sigma}^{\dagger}, \sigma=\uparrow, \downarrow$, are the familiar fermion operators, satisfy the hard-core boson relations

$$
\begin{aligned}
& \left(b_{j}^{+}(a)\right)^{2}=0, \quad\left[b_{j}(a), b_{k}^{+}(b)\right]=\delta_{a b} \delta_{j k}\left(1-2 b_{j}^{+}(a) b_{j}(a)\right), \\
& {\left[b_{j}(a), b_{k}(b)\right]=\left[b_{j}^{+}(a), b_{k}^{+}(b)\right]=0, \quad \text { for } k \neq j .}
\end{aligned}
$$

We can see from the Hamiltonian expression that the exchange interaction of Cooper pairs in one system depends on the number of Cooper pairs in the other system. For example, if in system (1), the level $j$ is empty and the level $k$ is occupied by one Cooper 
(1)

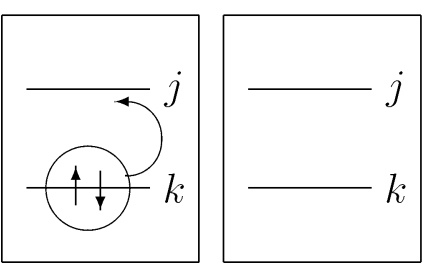

(1)

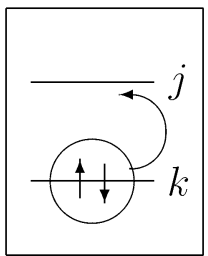

(2)

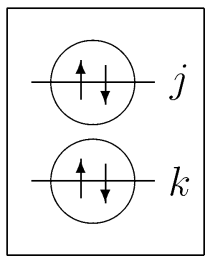

Fig. 1.

(1)

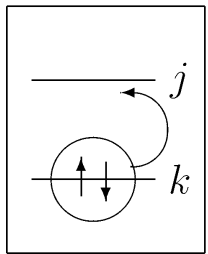

$(2)$

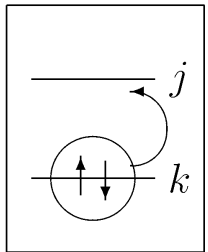

Fig. 2.

pair, just for certain configurations of system (2) it is possible that this Cooper pair in (1) scatters from level $k$ to $j$. This means that the Hamiltonian (2.1) presents naturally some "selection rules" for the scattering of states. We illustrate these configurations to indicate the possible pair scatterings in Fig. 1.

In addition, the double-pair scattering terms of the form (as shown in Fig. 2) are also present. What the above indicates is that besides the number of Cooper pairs being conserved in each system, the number of double pairs (to be more precise, the number of energy levels which are completely filled) is also conserved. This can be seen in each of the scattering processes depicted graphically above. In each case the scattering does not overall change the number of completely filled levels. There are further symmetries in the Hamiltonian. For example, there is a reflection symmetry which interchanges the labels 1 and 2 for the two BCS systems. This arises as a result of a global $s o(3) \oplus u(1)$ symmetry that the model possesses, which will be made more clear later. In that which follows we shall first discuss the integrability of the Hamiltonian (2.1) in the context of the QISM.

In order to built up a mechanism to construct an integrable $s u(4)$ pairing model, let us first recall the quantum $R$-matrix associated with the Lie algebra $s u(4)$, which acts in the tensor product of two 4-dimensional spaces $V \otimes V$ and can be written as

$$
R(\lambda)=\frac{(\lambda . I \otimes I+\eta P)}{(\lambda+\eta)} .
$$

Above $\lambda$ is the usual spectral parameter, $P$ is the permutation operator with matrix elements $P_{\alpha \beta, \gamma \delta}=\delta_{\alpha \delta} \delta_{\beta \gamma}, \alpha, \beta, \gamma, \delta=1,2,3,4$ and $\eta$ is the quasiclassical limit parameter; i.e.,

$$
\lim _{\eta \rightarrow 0} R(\lambda)=I \otimes I .
$$


It is known that this $R$-matrix satisfies the Yang-Baxter equation (YBE)

$$
R_{12}(\lambda-\mu) R_{13}(\lambda) R_{23}(\mu)=R_{23}(\mu) R_{13}(\lambda) R_{12}(\lambda-\mu) .
$$

The $R$-matrix may be viewed as the structural constants for the Yang-Baxter algebra generated by the monodromy matrix $T(\lambda)$, namely,

$$
R_{12}(\lambda-\mu) \stackrel{1}{T}(\lambda) \stackrel{2}{T}(\mu)=\stackrel{2}{T}(\mu) \stackrel{1}{T}(\lambda) R_{12}(\lambda-\mu) .
$$

Consequently, the $R$-matrix (2.3) allows us to construct a realization of the monodromy matrix through

$$
T(\lambda)=G_{0} R_{0 \Omega}\left(\lambda-\epsilon_{\Omega}\right) \cdots G_{0} R_{01}\left(\lambda-\epsilon_{1}\right) .
$$

Here the subscript 0 denotes the auxiliary space and $G$ satisfying

$$
[R, G \otimes G]=0,
$$

is a class of $c$-valued solutions of the YBE (2.4). As a consequence of the Yang-Baxter algebra (2.5), the transfer matrices $t(\lambda)=\operatorname{tr}_{0} T(\lambda)$ mutually commute for different values of the spectral parameter $\lambda$. This transfer matrix is the starting point in the construction of a $s u(4)$-type Gaudin Hamiltonian, from which we can obtain the $s u(4)$ pairing Hamiltonian, as will be shown below. For this purpose we make the following identification for the basis states

$$
\begin{aligned}
& |1\rangle=|0\rangle=\square- \\
& |2\rangle=b^{+}(1) b^{+}(2)|0\rangle=\uparrow \downarrow \uparrow \uparrow, \\
& |3\rangle=b^{+}(1)|0\rangle=\uparrow \downarrow+ \\
& |4\rangle=b^{+}(2)|0\rangle=-\stackrel{\uparrow}{\uparrow},
\end{aligned}
$$

and choose the $G$-matrix to be given by

$$
G \equiv \exp \left[\frac{2 \eta(1-n(1)-n(2))}{\Omega g}\right]=\left(\begin{array}{cccc}
\exp \left(\frac{2 \eta}{\Omega g}\right) & 0 & 0 & 0 \\
0 & \exp \left(\frac{-2 \eta}{\Omega g}\right) & 0 & 0 \\
0 & 0 & 1 & 0 \\
0 & 0 & 0 & 1
\end{array}\right)
$$

to construct the transfer matrix $t(\lambda)$. It can be verified that

$$
\begin{aligned}
t\left(\epsilon_{j}\right)= & \operatorname{tr}_{0}\left\{G_{0} R_{0 \Omega}\left(\epsilon_{j}-\epsilon_{\Omega}\right) \cdots G_{0} P_{0 j} \cdots G_{0} R_{01}\left(\epsilon_{j}-\epsilon_{1}\right)\right\} \\
= & G_{j} R_{j, j-1}\left(\epsilon_{j}-\epsilon_{j-1}\right) \cdots G_{j} R_{j 1}\left(\epsilon_{j}-\epsilon_{1}\right) \\
& \times G_{j} R_{j, \Omega}\left(\epsilon_{j}-\epsilon_{\Omega}\right) \cdots G_{j} R_{j, j+1}\left(\epsilon_{j}-\epsilon_{j+1}\right) G_{j}\left\{\operatorname{tr}_{0} P_{0 j}\right\} \\
= & G_{j} R_{j, j-1}\left(\epsilon_{j}-\epsilon_{j-1}\right) \cdots G_{j} R_{j 1}\left(\epsilon_{j}-\epsilon_{1}\right) \\
& \times G_{j} R_{j, \Omega}\left(\epsilon_{j}-\Omega\right) \cdots G_{j} R_{j, j+1}\left(\epsilon_{j}-\epsilon_{j+1}\right) G_{j} .
\end{aligned}
$$


Above $\operatorname{tr}_{0} P_{0 j}=1$. Next, taking the quasiclassical limit, we find

$$
\begin{aligned}
& \left.R_{j, k}(\lambda)\right|_{\eta \rightarrow 0}=I \otimes I+\eta r_{j, k}(\lambda)+\mathrm{O}\left(\eta^{2}\right), \\
& \left.G_{j}\right|_{\eta \rightarrow 0}=I+\frac{2 \eta}{\Omega g}\left(1-n_{j}(1)-n_{j}(2)\right)+\mathrm{O}\left(\eta^{2}\right),
\end{aligned}
$$

where $r_{j, k}(\lambda)=\frac{P_{j, k}-1}{\lambda}$. Thus it follows that

$$
\left.t\left(\epsilon_{j}\right)\right|_{\eta \rightarrow 0}=1+\eta\left(\tau_{j}-\sum_{\substack{k=1 \\ k \neq j}}^{\Omega} \frac{1}{\epsilon_{j}-\epsilon_{k}}\right)+\cdots,
$$

where

$$
\tau_{j}=\frac{2}{g}\left(1-n_{j}(1)-n_{j}(2)\right)+\sum_{\substack{k=1 \\ k \neq j}}^{\Omega} \frac{\sum_{\alpha, \beta}^{4} E_{j}^{\alpha \beta} E_{k}^{\beta \alpha}}{\epsilon_{j}-\epsilon_{k}} .
$$

Here $E^{\alpha \beta}=|\alpha\rangle\langle\beta|, \alpha, \beta=1, \ldots, 4$ are the Hubbard operators. An immediate consequence from the Yang-Baxter algebra (2.5) is that $\left[\tau_{j}, \tau_{k}\right]=0$. In addition, as a result of the $s o(3) \oplus u(1)$ symmetry mentioned earlier, it can be shown that there are extra conserved operators $K$ and $\chi$ such that

$$
\left[\tau_{j}, K\right]=\left[\tau_{j}, \chi\right]=[K, \chi]=0 .
$$

Above, $K$ is the Casimir operator of an so(3) subalgebra acting on the $\Omega$-fold tensor product

$$
K=\sum_{j, k}^{\Omega}\left(L_{j}^{+} L_{k}^{-}+L_{j}^{-} L_{k}^{+}+\frac{1}{2} L_{j}^{0} L_{k}^{0}\right),
$$

where $\left(L^{0}, L^{+}, L^{-}\right)$are the basis elements of this canonical so(3) subalgebra

$$
\begin{aligned}
& L^{+}=E^{34}=b^{+}(1) b(2), \\
& L^{-}=E^{43}=b^{+}(2) b(1), \\
& L^{0}=E^{33}-E^{44}=n(1)-n(2) .
\end{aligned}
$$

The $u(1)$ operator $\chi$ explicitly reads

$$
\chi=\sum_{j=1}^{\Omega}\left(E_{j}^{33}+E_{j}^{44}\right)=\sum_{j=1}^{\Omega}\left(n_{j}(1)-n_{j}(2)\right)^{2} .
$$

Any Hamiltonian which is defined in terms of the mutually commuting set of operators

$$
\left\{\tau_{j}, K, \chi\right\}
$$


will necessarily be integrable where the operators in (2.17) represent the constants of the motion. By making the following choice

$$
\begin{aligned}
H= & -g \sum_{j=1}^{\Omega} \epsilon_{j} \tau_{j}+\frac{g^{3}}{16} \sum_{j, k=1}^{\Omega} \tau_{j} \tau_{k}+\frac{3 g^{2}}{4} \sum_{j=1}^{\Omega} \tau_{j}+\frac{g}{2} K \\
& +\frac{g}{2} \chi(\chi-\Omega)+2 \sum_{j}^{\Omega} \epsilon_{j}+\frac{g \Omega^{2}}{4}-2 g \Omega,
\end{aligned}
$$

we produce the Hamiltonian (2.1). In order to determine the energy spectrum of this model, we will need to determine the eigenvalues of the conserved operators, to which we turn next.

\section{Bethe ansatz solutions}

Besides proving the integrability of the model, we can also obtain its exact solution from the algebraic Bethe ansatz for the standard $s u(4)$ vertex model constructed from the $R$-matrix (2.3). Employing the nested algebraic Bethe ansatz [15] we can obtain the eigenvalue of the transfer matrix (2.6) as

$$
\begin{aligned}
\Lambda\left(v,\left\{\lambda_{j}\right\}\left\{u_{i}\right\}\right)= & e^{\frac{2 \eta}{g}} \prod_{i=1}^{N} \frac{v-v_{i}-\eta}{v-v_{i}} \\
& +e^{-\frac{2 \eta}{g}} \prod_{i=1}^{\Omega} \frac{v-\epsilon_{i}-\frac{\eta}{2}}{v-\epsilon_{i}+\frac{\eta}{2}} \prod_{i=1}^{N} \frac{v-v_{i}+\eta}{v-v_{i}} \prod_{l=1}^{M} \frac{v-u_{l}-\frac{\eta}{2}}{v-u_{l}+\frac{\eta}{2}} \\
& +\prod_{i=1}^{\Omega} \frac{v-\epsilon_{i}-\frac{\eta}{2}}{v-\epsilon_{i}+\frac{\eta}{2}} \prod_{l=1}^{M} \frac{v-u_{l}+\frac{3 \eta}{2}}{v-u_{l}+\frac{\eta}{2}} \prod_{j=1}^{Q} \frac{v-w_{j}}{v-w_{j}+\eta} \\
& +\prod_{i=1}^{\Omega} \frac{v-\epsilon_{i}-\frac{\eta}{2}}{v-\epsilon_{i}+\frac{\eta}{2}} \prod_{j=1}^{Q} \frac{v-w_{j}+2 \eta}{v-w_{j}+\eta} .
\end{aligned}
$$

Above the parameters $v_{j}, u_{m}$ and $w_{k}$ satisfy the Bethe ansatz equations

$$
\begin{aligned}
& e^{-\frac{4 \eta}{g}} \prod_{i=1}^{\Omega} \frac{v_{j}-\epsilon_{i}-\frac{\eta}{2}}{v_{j}-\epsilon_{i}+\frac{\eta}{2}} \prod_{\substack{l=1 \\
l \neq j}}^{N} \frac{v_{j}-v_{l}+\eta}{v_{j}-v_{l}-\eta} \prod_{l=1}^{M} \frac{v_{j}-u_{l}-\frac{\eta}{2}}{v_{j}-u_{l}+\frac{\eta}{2}}=1, \\
& e^{-\frac{2 \eta}{g}} \prod_{i=1}^{N} \frac{u_{m}-v_{i}+\frac{\eta}{2}}{u_{m}-v_{i}-\frac{\eta}{2}}=\prod_{\substack{i=1 \\
i \neq m}}^{M} \frac{u_{m}-u_{i}+\eta}{u_{m}-u_{i}-\eta} \prod_{l=1}^{Q} \frac{u_{m}-w_{l}-\frac{\eta}{2}}{u_{m}-w_{l}+\frac{\eta}{2}}, \\
& \prod_{l=1}^{M} \frac{w_{k}-u_{l}+\frac{\eta}{2}}{w_{k}-u_{l}-\frac{\eta}{2}}=\prod_{\substack{l=1 \\
l \neq k}}^{Q} \frac{w_{k}-w_{l}+\eta}{w_{k}-w_{l}-\eta}
\end{aligned}
$$




$$
j=1, \ldots, N, \quad m=1, \ldots, M, \quad k=1, \ldots, Q .
$$

Defining $N(a)=\sum_{j=1}^{\Omega} n_{j}(a)$, we can readily determine that the quantum numbers $N, M$ and $Q$ are given by

$$
\begin{aligned}
& N=N(1)+N(2)-N(1) N(2), \\
& M=N(1)+N(2)-2 N(1) N(2), \\
& Q=N(2)-N(1) N(2) .
\end{aligned}
$$

The eigenvalues of the integrals of motion $\tau_{j}$ (2.13) can be obtained from the expansion of the eigenvalue of the transfer matrix (3.1) in the parameter $\eta$. Explicitly, the eigenvalues of $\tau_{j}$ are given by

$$
\Lambda_{j}=\frac{2}{g}+\sum_{l=1}^{N} \frac{1}{v_{l}-\epsilon_{j}}+\sum_{\substack{k=1 \\ k \neq j}}^{\Omega} \frac{1}{\epsilon_{j}-\epsilon_{k}},
$$

where the parameters satisfy the following equations

$$
\begin{aligned}
& \frac{4}{g}+\sum_{i=1}^{\Omega} \frac{1}{v_{j}-\epsilon_{i}}+\sum_{l=1}^{M} \frac{1}{v_{j}-u_{l}}=2 \sum_{\substack{l=1 \\
l \neq j}}^{N} \frac{1}{v_{j}-v_{l}}, \\
& \frac{2}{g}-\sum_{i=1}^{N} \frac{1}{u_{m}-v_{i}}-2 \sum_{\substack{l=1 \\
l \neq m}}^{M} \frac{1}{u_{l}-u_{m}}=\sum_{l=1}^{Q} \frac{1}{u_{m}-w_{l}}, \\
& \sum_{l=1}^{M} \frac{1}{w_{k}-u_{l}}=2 \sum_{\substack{l=1 \\
l \neq k}}^{Q} \frac{1}{w_{k}-w_{l}}, \\
& j=1, \ldots, N, \quad m=1, \ldots, M, \quad k=1, \ldots, Q .
\end{aligned}
$$

We will also need the eigenvalues of the operators $K$ and $\chi$. Through use of (2.15), (2.16), (3.2) we find that $\chi$ has eigenvalue $M$ while the eigenvalues of $K$ are

$$
\frac{1}{2}(M-2 Q)(M-2 Q+2) \text {. }
$$

Finally, utilizing (3.3) and noting the following identities which can be derived from (3.4):

$$
\begin{aligned}
& \sum_{k=1}^{Q} \sum_{l=1}^{M} \frac{1}{w_{k}-u_{l}}=2 \sum_{k=1}^{Q} \sum_{l=1}^{Q} \frac{1}{w_{k}-w_{l}}=0, \\
& \sum_{m=1}^{M} \sum_{i=1}^{N} \frac{1}{u_{m}-v_{i}}=\frac{2 M}{g}
\end{aligned}
$$




$$
\begin{aligned}
& \sum_{j=1}^{N} \sum_{i=1}^{\Omega} \frac{1}{v_{j}-\epsilon_{i}}=-\frac{2(2 N-M)}{g}, \\
& \sum_{k=1}^{Q} \sum_{l=1}^{M} \frac{u_{l}}{u_{l}-w_{k}}-\sum_{k=1}^{Q} \sum_{l=1}^{M} \frac{w_{k}}{u_{l}-w_{k}}=M Q, \\
& \sum_{m=1}^{M} \sum_{i=1}^{N} \frac{v_{i}}{v_{i}-u_{m}}-\sum_{m=1}^{M} \sum_{i=1}^{N} \frac{u_{m}}{v_{i}-u_{m}}=M N, \\
& \sum_{j=1}^{N} \sum_{i=1}^{\Omega} \frac{\epsilon_{i}}{\epsilon_{i}-v_{j}}-\sum_{j=1}^{N} \sum_{i=1}^{\Omega} \frac{v_{j}}{\epsilon_{i}-v_{j}}=N \Omega, \\
& \sum_{k=1}^{Q} \sum_{l=1}^{M} \frac{w_{k}}{w_{k}-u_{l}}=Q(Q-1), \\
& -\frac{2}{g} \sum_{m=1}^{M} u_{m}+\sum_{m=1}^{M} \sum_{i=1}^{N} \frac{u_{m}}{u_{m}-v_{i}}+\sum_{m=1}^{M} \sum_{l=1}^{Q} \frac{u_{m}}{u_{m}-w_{l}}=M(M-1), \\
& \frac{4}{g} \sum_{j=1}^{N} v_{j}-\sum_{j=1}^{N} \sum_{i=1}^{\Omega} \frac{v_{j}}{\epsilon_{i}-v_{j}}+\sum_{j=1}^{N} \sum_{l=1}^{M} \frac{v_{j}}{v_{j}-u_{l}}=N(N-1),
\end{aligned}
$$

we can present from the relation (2.18) the eigenvalue of the Hamiltonian (2.1) as

$$
E=4 \sum_{i=1}^{N} v_{i}-2 \sum_{m=1}^{M} u_{m}-g(2 N-3 M) .
$$

Let us make some small remarks about the degeneracies of the spectrum. Though the eigenstates of the Hamiltonian have not been made explicit here, it can be deduced by the standard arguments (e.g., [16]) that each is a highest weight state with respect to the so(3) symmetry algebra (2.15). In particular, the highest weight which is given by the eigenvalue of the operator $L^{0}$ is $M-2 Q$, so we can conclude that the multiplet generated by (2.15) acting on this highest weight state has dimension $M-2 Q+1$. Therefore for each solution of (3.4) with given $N, M$ and $Q$, the corresponding energy level has degeneracy $M-2 Q+1$.

\section{Asymptotic solutions}

As the Bethe ansatz equations (3.4) take the form of coupled non-linear equations it is unlikely to find analytic solutions, and one tends to resort to numerical analysis. It is however possible to conduct an asymptotic analysis for small values of the coupling parameter $g$. Below we undertake this for the ground state of the system and some elementary excitations. 
(1)

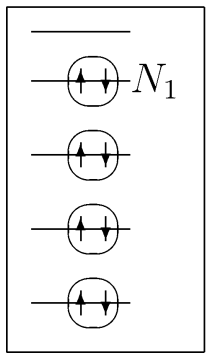

$(2)$

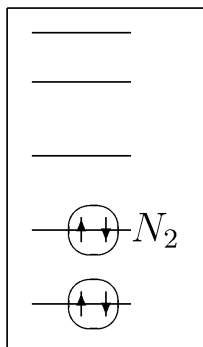

Fig. 3.

For the ground state, we consider first the case $g=0$. Letting $N_{1}$ and $N_{2}$ denote the number of Cooper pairs in each system, then it is clear that the ground state corresponds to filling the Fermi sea, which is illustrated in Fig. 3, where without loss of generality we assume that $N_{1}>N_{2}$. For small $g \neq 0$ we see that the ground state will be described by a solution of (3.4) with $N=N_{1}, M=N_{1}-N_{2}, Q=0$.

Thus the Bethe equations (3.4) reduce to two levels for the parameters $v_{j}$ and $u_{m}$. For a small $g>0$ it is appropriate to consider the asymptotic solution

$$
\begin{aligned}
& v_{j}=\epsilon_{j}+g \delta_{j}+g^{2} \sigma_{j}, \quad j=1, \ldots, N_{1}, \\
& u_{m}=\epsilon_{N_{1}-m+1}+g \alpha_{m}+g^{2} \beta_{m}, \quad m=1, \ldots, N_{1}-N_{2} .
\end{aligned}
$$

Substituting these into the Bethe equations (3.4) with the configuration $N=N_{1}, M=$ $N_{1}-N_{2}, Q=0$, one can find that

$$
\begin{aligned}
& v_{j} \approx \epsilon_{j}-\frac{g}{4}+\frac{g^{2}}{16}\left[\sum_{i=N_{1}+1}^{\Omega} \frac{1}{\epsilon_{j}-\epsilon_{i}}-\sum_{\substack{l=1 \\
l \neq j}}^{N_{2}} \frac{1}{\epsilon_{j}-\epsilon_{l}}\right], \quad j \leqslant N_{2}, \\
& v_{j} \approx \epsilon_{j}-\frac{g}{2}+\frac{g^{2}}{4}\left[\sum_{i=N_{1}+1}^{\Omega} \frac{1}{\epsilon_{j}-\epsilon_{i}}-\sum_{\substack{l=N_{2}+1 \\
l \neq j}}^{N_{1}} \frac{1}{\epsilon_{j}-\epsilon_{l}}\right], \quad j>N_{2}, \\
& u_{m} \approx \epsilon_{N_{1}-m+1}+\frac{g^{2}}{4}\left[\sum_{i=N_{1}+1}^{\Omega} \frac{1}{\epsilon_{N_{1}-m+1}-\epsilon_{i}}+\sum_{i=1}^{N_{2}} \frac{1}{\epsilon_{N_{1}-m+1}-\epsilon_{i}}\right. \\
& \left.-\sum_{\substack{l=N_{2}+1 \\
l \neq N_{1}-m+1}}^{N_{1}} \frac{2}{\epsilon_{N_{1}-m+1}-\epsilon_{l}}\right], \quad m=1, \ldots, N_{1}-N_{2} .
\end{aligned}
$$

The asymptotic ground state energy is deduced to be given by 


$$
\begin{aligned}
E_{0} \approx & 4 \sum_{j=1}^{N_{1}} \epsilon_{j}-2 \sum_{l=N_{2}+1}^{N_{1}} \epsilon_{l}-\left(N_{1}+2 N_{2}\right) g \\
+ & \frac{g^{2}}{4}\left[\sum_{j=1}^{N_{2}} \sum_{i=N_{1}+1}^{\Omega} \frac{1}{\epsilon_{j}-\epsilon_{i}}+\sum_{j=N_{2}+1}^{N_{1}}\left(\sum_{i=N_{1}+1}^{\Omega} \frac{2}{\epsilon_{j}-\epsilon_{i}}-\sum_{i=1}^{N_{2}} \frac{2}{\epsilon_{j}-\epsilon_{i}}\right)\right] .
\end{aligned}
$$

It is important to point out that from the above ground state energy we can infer some results about the asymptotic behaviour of zero temperature correlation functions. Specifically, by employing the Hellmann-Feynman theorem we have that

$$
\left\langle n_{i}(1)+n_{i}(2)\right\rangle=\frac{1}{2} \frac{\partial E_{0}}{\partial \epsilon_{i}} .
$$

The result obtained is

$$
\begin{aligned}
& \left\langle n_{i}(1)+n_{i}(2)\right\rangle \\
& \approx \frac{g^{2}}{8}\left(\sum_{j=1}^{N_{2}} \frac{1}{\left(\epsilon_{j}-\epsilon_{i}\right)^{2}}+\sum_{j=N_{2}+1}^{N_{1}} \frac{2}{\left(\epsilon_{j}-\epsilon_{i}\right)^{2}}\right), \quad \text { for } i>N_{1}, \\
& \left\langle n_{i}(1)+n_{i}(2)\right\rangle \\
& \quad \approx 1-\frac{g^{2}}{8}\left(\sum_{j=N_{1}+1}^{\Omega} \frac{2}{\left(\epsilon_{j}-\epsilon_{i}\right)^{2}}-\sum_{j=1}^{N_{2}} \frac{2}{\left(\epsilon_{j}-\epsilon_{i}\right)^{2}}\right), \quad \text { for } N_{1} \geqslant i>N_{2}, \\
& \left\langle n_{i}(1)+n_{i}(2)\right\rangle \\
& \quad \approx 2-\frac{g^{2}}{8}\left(\sum_{j=N_{1}+1}^{\Omega} \frac{1}{\left(\epsilon_{j}-\epsilon_{i}\right)^{2}}+\sum_{j=N_{2}+1}^{N_{1}} \frac{2}{\left(\epsilon_{j}-\epsilon_{i}\right)^{2}}\right), \quad \text { for } i \leqslant N_{2} .
\end{aligned}
$$

Next let us consider a possible excitation which can be obtained by breaking one Cooper pair in BCS(2). In the $g=0$ case, the excited state is depicted in Fig. 4.

For non-zero $g$, we choose $N=N_{1}-2, M=N_{1}-N_{2}-1$ and block the levels with energy $\epsilon_{N_{2}}, \epsilon_{N_{2}+1}$. From the asymptotic solutions (4.3)-(4.5), we obtain the excitation energy

$$
\begin{aligned}
E_{1} \approx & 4 \sum_{j=1}^{N_{1}} \epsilon_{j}-2 \sum_{l=N_{2}+2}^{N_{1}} \epsilon_{l}-\epsilon_{N_{2}}-\epsilon_{N_{2}+1}-\left(N_{1}+2 N_{2}-4\right) g \\
+ & \frac{g^{2}}{4}\left[\sum_{j=1}^{N_{2}-1} \sum_{i=N_{1}+1}^{\Omega} \frac{1}{\epsilon_{j}-\epsilon_{i}}+\sum_{j=N_{2}+2}^{N_{1}}\left(\sum_{i=N_{1}+1}^{\Omega} \frac{2}{\epsilon_{j}-\epsilon_{i}}-\sum_{i=1}^{N_{2}-1} \frac{2}{\epsilon_{j}-\epsilon_{i}}\right)\right] .
\end{aligned}
$$

Therefore the gap obtained through the breaking a Cooper pair in BCS(2) is given by 
(1)

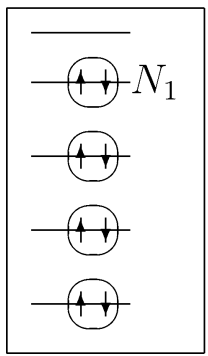

Fig. 4.

(1)

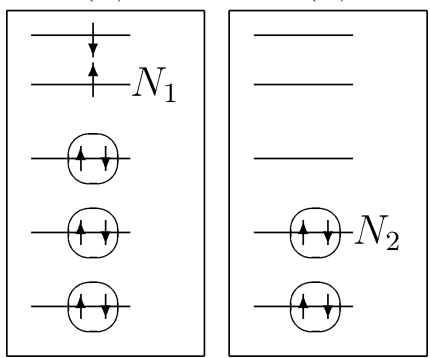

$(2)$

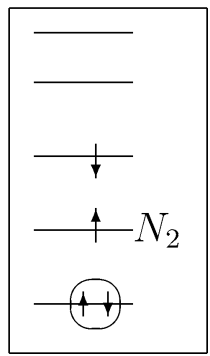

Fig. 5.

$$
\begin{aligned}
\Delta_{1} \approx \epsilon_{N_{2}+1}-\epsilon_{N_{2}}+4 g+\frac{g^{2}}{4}[ & \sum_{i=N_{1}+1}^{\Omega} \frac{1}{\epsilon_{i}-\epsilon_{N_{2}}}+\sum_{i=N_{1}+1}^{\Omega} \frac{2}{\epsilon_{i}-\epsilon_{N_{2}+1}} \\
& \left.+\sum_{j=N_{2}+2}^{N_{1}} \frac{2}{\epsilon_{j}-\epsilon_{N_{2}}}+\sum_{i=1}^{N_{2}} \frac{2}{\epsilon_{N_{2}+1}-\epsilon_{i}}\right] .
\end{aligned}
$$

Another possibility for an excitation at $g=0$ is to break the Cooper pair at level $\epsilon_{N_{1}}$ in BCS(1) (see Fig. 5). For $g \neq 0$, the configuration should be accommodated as $N=N_{1}-1, M=N_{1}-N_{2}-1$ with the levels $\epsilon_{N_{1}}, \epsilon_{N_{1}+1}$ blocked.

We find the gap obtained in this case to be given by

$$
\begin{aligned}
\Delta_{2} \approx \epsilon_{N_{1}+1}-\epsilon_{N_{1}}+g+\frac{g^{2}}{4}[ & \sum_{j=1}^{N_{2}} \frac{1}{\epsilon_{N_{1}+1}-\epsilon_{j}}+\sum_{i=1}^{N_{2}} \frac{2}{\epsilon_{N_{1}}-\epsilon_{i}} \\
& \left.+\sum_{j=N_{2}+1}^{N_{1}-1} \frac{2}{\epsilon_{N_{1}+1}-\epsilon_{j}}+\sum_{i=N_{1}+1}^{\Omega} \frac{2}{\epsilon_{i}-\epsilon_{N_{1}}}\right] .
\end{aligned}
$$

For the above asymptotic analysis to be valid, we require that the coefficients of the terms in $g^{2}$ must be much smaller than $g^{-1}$, imposing a stringent constraint on $g$. In each case this will depend explicitly on $\Omega, N_{1}, N_{2}$ and the distribution of the single particle energy levels $\epsilon_{i}$. For the case of the usual BCS model such a constraint was studied in [18]. 


\section{Conclusion}

To summarize, we have constructed an integrable pairing Hamiltonian based on the $s u(4)$ Lie algebra. This model can be interpreted as describing two coupled BCS systems of different types, such as for protons and neutrons in a nuclear system. The Bethe ansatz equations and the energies of the model have been calculated. For small values of the coupling parameter $g$, we asymptotically analyzed the ground state and elementary excitations, and the expectation values for the occupation numbers. An open problem that we will address in the future is the exact calculation of form factors and correlation functions using the techniques developed by Babujian et al. in [17].

\section{Acknowledgements}

X.-W.G. gratefully acknowledges the Centre for Mathematical Physics, the University of the Queensland for kind hospitality and FAPERGS (Fundação de Amparo ã Pesquisa do Estado do Rio Grande do Sul) for financial support. A.F. would like to acknowledge M. Karowski and H. Babujian for discussions and the group of Theoretical Physics of the FU-Berlin for their kind hospitality. She also thanks DAAD (Deutsche Akademischer Austauschdienst) and FAPERGS for financial support. H.-Q.Z. and J.L. thank the Australian Research Council.

\section{References}

[1] R.J. Baxter, Exactly Solved Models in Statistical Mechanics, Academic Press, San Diego, 1982.

[2] F.H.L. Essler, V.E. Korepin, Phys. Rev. B 46 (1992) 9147;

A. Foerster, M. Karowski, Phys. Rev. B 46 (1992) 9234;

A. Foerster, M. Karowski, Nucl. Phys. B 396 (1993) 611.

[3] B.S. Shastry, Phys. Rev. Lett. 56 (1986) 1529;

B.S. Shastry, Phys. Rev. Lett. 56 (1986) 2453;

M.J. Martins, P.B. Ramos, Nucl. Phys. B 522 (1998) 413.

[4] M. Gaudin, J. Physique 37 (1976) 1087;

H. Babujian, J. Phys. A 26 (1993) 6981.

[5] R.W. Richardson, Phys. Lett. 3 (1963) 277;

R.W. Richardson, Phys. Lett. 5 (1963) 82;

R.W. Richardson, N. Sherman, Nucl. Phys. 52 (1964) 221;

R.W. Richardson, N. Sherman, Nucl. Phys. 52 (1964) 253.

[6] M.C. Cambiaggio, A.M.F. Rivas, M. Saraceno, Nucl. Phys. A 624 (1997) 157.

[7] J. von Delft, D.C. Ralph, Phys. Rep. 345 (2001) 61;

J. von Delft, Ann. Phys. (Leipzig) 10 (2001) 219.

[8] D.C. Ralph, C.T. Black, M. Tinkham, Phys. Rev. Lett. 76 (1996) 688;

D.C. Ralph, C.T. Black, M. Tinkham, Phys. Rev. Lett. 78 (1997) 4087.

[9] H.-Q. Zhou, J. Links, R.H. McKenzie, M.D. Gould, Phys. Rev. B 65 (2002) 060502(R).

[10] J. von Delft, R. Poghossian, cond-mat/0106405.

[11] M. Asorey, F. Falceto, G. Sierra, Nucl. Phys. B 622 (2002) 593.

[12] J. Links, H.-Q. Zhou, M.D. Gould, R.H. McKenzie, J. Phys. A 35 (2002) 6459.

[13] R.W. Richardson, Phys. Rev. 144 (1966) 874;

R.W. Richardson, Phys. Rev. 154 (1967) 1007. 
[14] J. Links, H.-Q. Zhou, R.H. McKenzie, M.D. Gould, cond-mat/0110105, Int. J. Mod. Phys. B, in press; J. Links, K.E. Hibberd, Int. J. Mod. Phys. B 16 (2002) 2009.

[15] B. Sutherland, Phys. Rev. B 12 (1975) 3795;

O. Babelon, H.J. de Vega, C.M. Viallet, Nucl. Phys. B 200 (1982) 266.

[16] A.N. Kirillov, J. Sov. Math. 36 (1987) 115.

[17] H. Babujian, A. Fring, M. Karowski, Form factors of the $S U(N)$ model, in preparation.

[18] M. Schechter, Y. Imry, Y. Levinson, J. von Delft, Phys. Rev. B 63 (2001) 214518. 